\title{
Multifractality of the Feigenbaum attractor and fractional derivatives
}

\author{
U. Frisch ${ }^{1}$, K. Khanin ${ }^{1-4}$ and T. Matsumoto ${ }^{1,5}$ \\ ${ }^{1}$ Observatoire de la Côte d'Azur, B.P. 4229, 06304 Nice Cedex 4, France \\ ${ }^{2}$ Issac Newton Institute for Mathematical Sciences, 20 Clarkson Road, Cambridge CB3 0EH, U.K. \\ ${ }^{3}$ Dept. of Mathematics, Heriot-Watt University, Edinburgh EH14 4AS, U.K. \\ ${ }^{4}$ Landau Institute for Theoretical Physics, Kosygina Str. 2, Moscow 117332, Russia. \\ ${ }^{5}$ Dept. of Physics, Kyoto University, Kitashirakawa Oiwakecho Sakyo-ku Kyoto 6068502, Japan.
}

November 20, 2018

J. Stat. Phys. in press

\begin{abstract}
It is shown that fractional derivatives of the (integrated) invariant measure of the Feigenbaum map at the onset of chaos have power-law tails in their cumulative distributions, whose exponents can be related to the spectrum of singularities $f(\alpha)$. This is a new way of characterizing multifractality in dynamical systems, so far applied only to multifractal random functions (Frisch and Matsumoto (J. Stat. Phys. 108:1181, 2002)). The relation between the thermodynamic approach (Vul, Sinai and Khanin (Russian Math. Surveys 39:1, 1984)) and that based on singularities of the invariant measures is also examined. The theory for fractional derivatives is developed from a heuristic point view and tested by very accurate simulations.
\end{abstract}

Keywords: chaotic dynamics, multifractals, thermodynamic formalism.

\section{Introduction}

Recently a new method for analyzing multifractal functions $u(x)$ was introduced [1]. It exploits the fact that the fractional derivative of order $a$ (denoted here by $D^{a}$ ) of $u(x)$ has, for a suitable range of $a$, a power-law tail in its cumulative probability

$$
\operatorname{Prob}\left\{\left|D^{a} u\right|>\xi\right\} \propto \xi^{-p_{\star}}, \quad \xi \rightarrow \infty .
$$

The exponent $p_{\star}(a)$ is the unique solution of the equation

$$
\zeta_{p_{\star}}=p_{\star}(a) a,
$$

where $\zeta_{p}$ is the scaling exponent associated to the behavior at small separations $l$ of the structure function of order $p$, i.e. $\left\langle|u(x+l)-u(x)|^{p}\right\rangle \sim|l|^{\zeta_{p}}$. It was also shown that the actual observability of the power-law tail when multifractality is restricted to a finite range of scales is controlled by 
how much $\zeta_{p}$ departs from linear dependence on $p$. The larger this departure the easier it is to observe multifractality.

So far the theory of such power-law tails has been developed only for synthetic random functions, in particular the random multiplicative process [2] for which Kesten-type maps [3] and large deviations theory can be used.

It is our purpose here to test the fractional derivative method for invariant measures of dissipative dynamical systems, in particular for the Feigenbaum invariant measure which appears at the accumulation point of the period doubling cascade where the orbit has period $2^{\infty}$ [4, 5]. Its multifractality was proven rigorously in Ref. 6] using a thermodynamic formalism. For the Feigenbaum measure all scaling exponents can be determined with arbitrary accuracy.

There is an important difference in the way one processes functions and invariant measures to determine their multifractal properties and in particular the spectrum of singularities, usually denoted $D(h)$ for functions [7] and $f(\alpha)$ for measures [8, 9]. For a function $u(x)$ one uses the moments or the PDFs of the increments $u(x+l)-u(x)$ to determine the scaling exponents, whereas for an invariant measure $\mu_{0}$ one works with integrals over intervals or boxes of different sizes. In the one-dimensional case the two approaches become equivalent by introducing the cumulative distribution function

$$
u(x) \equiv \int_{-\infty}^{x} \mu_{0}(d x) .
$$

Hence we shall apply the fractional derivative method to the integral of the invariant measure.

The organization of the paper is the following. Section 2 is devoted to the thermodynamic formalism for the Feigenbaum attractor. In Section 2.1, we recall the method used in Ref. 6]. In Section 2.2 we show how this formalism, based on the study of the geometrical properties of the attractor, is actually connected to the standard multifractal formalism which focusses on the statistical properties of the invariant measure [8, 9]. To the best of our knowledge the exact relation between the two formalisms is discussed here for the first time. Then, in Section [2.3] we calculate numerically the free energy and accordingly the scaling exponents $\zeta_{p}$ for the integral of the invariant measure; this is done by a very accurate transfer-matrix-based method. Fractional derivatives are discussed in Section [3. In Section 3.1 we briefly recall the phenomenology of power-law tails in the distribution of fractional derivatives and the limits on observability. The fractional derivative analysis of the Feigenbaum measure is presented in Section 3.2 Concluding remarks are made in Section 4.

\section{Thermodynamic Formalism for the Feigenbaum Attractor}

\subsection{Thermodynamic formalism}

In this section we give a brief description of the thermodynamic formalism for the invariant measure of the Feigenbaum map (see Ref. [6] for the mathematical details) and show how one can use it in order to study the multifractal properties of the Hölder exponents.

By Feigenbaum attractor we understand the attractor of the one-dimensional mapping $g$ : $[0,1] \rightarrow[0,1]$, where $g(x)$ is the solution of the Feigenbaum-Cvitanović doubling equation:

$$
g(x)=-\frac{1}{\gamma} g(g(\gamma x)), \quad g(0)=1, \quad \gamma=\frac{1}{\alpha}=-g(1) .
$$




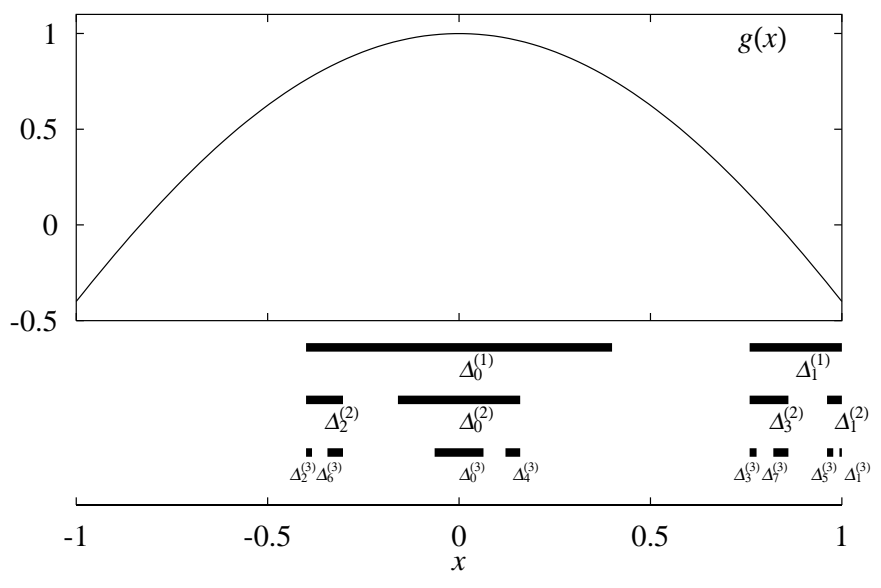

Figure 1: The Feigenbaum-Cvitanović map $g(x)$ and the first three levels of the partitions $\Delta_{j}^{(n)}$. For $g(x)$ we used the expansion (5), introduced in Ref. [10] up to $x^{32}$.

Equation (4) is known to have the unique solution in the class of smooth unimodal maps (that is, maps having one critical point) with a non-degenerate maximum. This solution is called the Feigenbaum map. It is holomorphic in some complex neighborhood of $[-1,1]$ and the first few terms in the power series expansion are [10]

$$
g(x)=1-1.5276 \ldots x^{2}+0.1048 \ldots x^{4}+0.0267 \ldots x^{6}-0.003527 \ldots x^{8}+\ldots
$$

The value of the universal constant $\gamma$ which is the inverse of the Feigenbaum scaling constant $\alpha$ is approximately equal to 0.3995 . An attractor $A$ for the map $g$ can be constructed in the following way. For each $n \geq 1$ define a collection of intervals of $n$-th level:

$$
\begin{aligned}
& \Delta_{0}^{(n)}=\left[-\gamma^{n}, \gamma^{n}\right], \\
& \Delta_{i}^{(n)}=g^{(i)}\left(\Delta_{0}^{(n)}\right) \equiv \underbrace{g \circ g \circ \cdots \circ g}_{i}\left(\Delta_{0}^{(n)}\right) \quad\left(1 \leq i \leq 2^{n}-1\right) .
\end{aligned}
$$

The following properties of the intervals $\Delta_{i}^{(n)}$ are easy consequences of the doubling equation (41): (a) Intervals $\Delta_{i}^{(n)}, 0 \leq i \leq 2^{n}-1$ are pairwise disjoint. (b) $g^{\left(2^{n}\right)} \Delta_{0}^{(n)} \subset \Delta_{0}^{(n)}$. (c) Each interval of $n$-th level $\Delta_{i}^{(n)}$ contains exactly two intervals of $(n+1)$-th level, $\Delta_{i}^{(n+1)}$ and $\Delta_{i+2^{n}}^{(n+1)}$. (d) $2 \gamma^{2 n} \leq\left|\Delta_{i}^{(n)}\right| \leq 2 \gamma^{n}$, where $|\cdot|$ denotes the length of the interval. The first three levels of the intervals are shown in Fig. 1].

The properties above imply that it is natural to use a dyadic representation for the intervals $\Delta_{i}^{(n)}$. Let $i=\sum_{j=0}^{n-1} \epsilon_{j} 2^{i}$, where $\epsilon_{j}=0,1$. Then we can use a sequence $\left(\epsilon_{0}, \ldots, \epsilon_{n-1}\right)$ as a symbolic coding for intervals $\Delta_{i}^{(n)}: \Delta_{i}^{(n)}=\Delta_{\epsilon_{0}, \ldots, \epsilon_{n-1}}^{(n)}$. Now we can define the Feigenbaum attractor

$$
A=\bigcap_{n \geq 1} \bigcup_{i=0}^{2^{n}-1} \Delta_{i}^{(n)}
$$


The set $A$ is isomorphic to the set of all infinite dyadic sequences $\left(\epsilon_{0}, \epsilon_{1}, \ldots, \epsilon_{n}, \ldots\right)$. Such sequences can be considered as a symbolic coordinate system on $A$. In this new coordinate system the map $g$ acts as the dyadic addition of the sequence $(1,0, \ldots, 0, \ldots)$. Notice that topologically $A$ is a Cantor set. It is easy to see that $A$ is indeed an attractor for all but countably many initial points $x \in[-1,1]: \operatorname{dist}\left(g^{(m)}(x), A\right) \rightarrow 0$ as $m \rightarrow \infty$. The exceptional set of initial points consists of all unstable periodic orbits and their preimages.

As we have seen above, all intervals $\Delta_{i}^{(n)}$ have exponentially small lengths but the exponent varies from $2 \gamma^{n}$ to $\gamma^{2 n}$. Notice that exponents $\ln \left|\Delta_{i}^{(n)}\right|$ give all possible scalings of the fractal set $A$. The basic ingredient which is needed for the multifractal analysis is the control over the spectrum of possible scalings corresponding to exponents, i.e. $\ln \left|\Delta_{i}^{(n)}\right|$. Such control can be achieved with the help of the thermodynamic formalism. The thermodynamic formalism which was constructed in Ref. [6] is based on the Gibbsian description for the lengths of the intervals $\Delta_{i}^{(n)}$. It is shown in Ref. [6] that there exists a function $U\left(\epsilon^{(1)}, \epsilon^{(2)}, \ldots, \epsilon^{(n)}, \ldots\right)$ (thermodynamic potential) which is defined on all infinite dyadic sequences such that:

I. There exists a constant $C>0$ for which

$$
\frac{1}{C} \leq-U\left(\epsilon^{(1)}, \epsilon^{(2)}, \ldots, \epsilon^{(n)}, \ldots\right) \leq C .
$$

II. For any two dyadic sequences

$$
\left(\epsilon^{(1)}, \epsilon^{(2)}, \ldots, \epsilon^{(n)}, \bar{\epsilon}^{(n+1)}, \bar{\epsilon}^{(n+2)}, \ldots\right),\left(\epsilon^{(1)}, \epsilon^{(2)}, \ldots, \epsilon^{(n)}, \tilde{\epsilon}^{(n+1)}, \tilde{\epsilon}^{(n+2)}, \ldots\right)
$$

which coincide on the first $n$ positions

$$
\left|U\left(\epsilon^{(1)}, \epsilon^{(2)}, \ldots, \epsilon^{(n)}, \bar{\epsilon}^{(n+1)}, \bar{\epsilon}^{(n+2)}, \ldots\right)-U\left(\epsilon^{(1)}, \epsilon^{(2)}, \ldots, \epsilon^{(n)}, \tilde{\epsilon}^{(n+1)}, \tilde{\epsilon}^{(n+2)}, \ldots\right)\right| \leq C(2 \gamma)^{n} .
$$

III. For any $\Delta_{\epsilon_{0}, \ldots, \epsilon_{n-1}}^{(n)}$ with $\epsilon_{0}=1$

$$
\exp \left[-C(2 \gamma)^{\frac{n}{3}}\right] \leq \frac{\left|\Delta_{\epsilon_{0}, \ldots, \epsilon_{n-1}}^{(n)}\right|}{\left|\Delta_{\epsilon_{0}, \ldots, \epsilon_{n-2}}^{(n-1)}\right|} \exp \left(-U\left(\epsilon_{n-1}, \ldots, \epsilon_{1}, 1,0, \ldots, 0, \ldots\right)\right) \leq \exp \left[C(2 \gamma)^{\frac{n}{3}}\right]
$$

It immediately follows from (10) that for $C_{1}=\exp \left[\frac{C}{1-(2 \gamma)^{\frac{1}{3}}}\right]$

$$
\frac{1}{C_{1}} \leq \frac{\left|\Delta_{1, \epsilon_{1}, \ldots, \epsilon_{n-1}}^{(n)}\right|}{\exp \left[\sum_{s=0}^{n-1} U\left(\epsilon_{s}, \epsilon_{s-1}, \ldots, \epsilon_{1}, 1,0, \ldots, 0, \ldots\right)\right]} \leq C_{1}
$$

The condition $\epsilon_{0}=1$ corresponding to intervals $\Delta_{i}^{(n)}$ with odd $i$ 's plays only a technical role and it is not essential for our further analysis since the odd intervals contain information about the lengths of the even ones. Indeed, it is very easy to see that for every odd $i$ the intervals $\Delta_{i}^{(n)}$ and $\Delta_{i+1}^{(n)}$ have lengths of the same order.

We next introduce a parameter $\beta$ (inverse temperature) and define the partition function

$$
Z_{n}(\beta)=\sum_{\epsilon_{n-1}, \ldots, \epsilon_{0}=0,1} \exp \left[\beta \sum_{s=0}^{n-1} U\left(\epsilon_{s}, \epsilon_{s-1}, \ldots, \epsilon_{1}, 1,0, \ldots, 0, \ldots\right)\right]
$$


and the free energy

$$
F_{n}(\beta)=\frac{\ln Z_{n}(\beta)}{n}, \quad F(\beta)=\lim _{n \rightarrow \infty} F_{n}(\beta) .
$$

It immediately follows from (12) and (13) that

$$
\sum_{i=0}^{2^{n}-1}\left|\Delta_{i}^{(n)}\right|^{\beta} \sim \exp [n F(\beta)]
$$

In the thermodynamic limit $n \rightarrow \infty$ the probability distributions

$$
\nu_{\beta}^{(n)}\left(\epsilon_{n-1}, \epsilon_{n-2}, \ldots, \epsilon_{1}\right)=\frac{1}{Z_{n}(\beta)} \exp \left[\beta \sum_{s=0}^{n-1} U\left(\epsilon_{s}, \epsilon_{s-1}, \ldots, \epsilon_{1}, 1,0, \ldots, 0, \ldots\right)\right]
$$

tend to a limiting distribution $\nu_{\beta}$ which can be considered as a Gibbs measure with the potential $U$, inverse temperature $\beta$ and the boundary condition $\epsilon_{0}=1, \epsilon_{-i}=0, i \in \mathbb{N}$. This Gibbs distribution generates the probability measure on $A_{+}=A \cap \Delta_{1}^{(1)}$ which is the part of the whole attractor $A$ corresponding to intervals $\Delta_{i}^{(n)}$ with odd numbers $i$. We shall denote this Gibbs measure on $A_{+}$by $\mu_{\beta}$. Notice that $\beta=0$ corresponds to a unique invariant measure and $\beta=1$ gives a conditional distribution corresponding to Lebesgue measure on $[-1,1]$. The free energy $F(\beta)$ contains all information about the multifractal properties of the Feigenbaum attractor. Notice that the thermodynamic formalism leads to one-dimensional statistical mechanics with exponential decay of interactions and hence without phase transitions. This implies that $F(\beta)$ is a smooth function. In fact it is holomorphic in some complex neighborhood of the real axis. Denote

$$
H\left(\epsilon_{n-1}, \ldots, \epsilon_{1}\right)=\sum_{s=0}^{n-1} U\left(\epsilon_{s}, \epsilon_{s-1}, \ldots, \epsilon_{1}, 1,0, \ldots\right)
$$

Using relations

$$
\begin{aligned}
F_{n}^{\prime}(\beta) & =\frac{1}{Z_{n}(\beta)} \sum_{\epsilon_{n-1}, \ldots, \epsilon_{0}=0,1} \frac{1}{n} H\left(\epsilon_{n-1}, \ldots, \epsilon_{1}\right) \exp \left[\beta H\left(\epsilon_{n-1}, \ldots, \epsilon_{1}\right)\right] \\
& =\left\langle\frac{\ln \left|\Delta_{1, \epsilon_{1}, \ldots, \epsilon_{n-1}}^{(n)}\right|}{n}\right\rangle_{\nu_{\beta}^{(n)}}+O\left(\frac{1}{n}\right)
\end{aligned}
$$

and

$$
\begin{aligned}
F_{n}^{\prime \prime}(\beta) & =\frac{1}{Z_{n}(\beta)} \sum_{\epsilon_{n-1}, \ldots, \epsilon_{0}=0,1}\left(\frac{1}{n} H\left(\epsilon_{n-1}, \ldots, \epsilon_{1}\right)\right)^{2} \exp \left[\beta H\left(\epsilon_{n-1}, \ldots, \epsilon_{1}\right)\right] \\
& -\left(\frac{1}{Z_{n}(\beta)} \sum_{\epsilon_{n-1}, \ldots, \epsilon_{0}=0,1} \frac{1}{n} H\left(\epsilon_{n-1}, \ldots, \epsilon_{1}\right) \exp \left[\beta H\left(\epsilon_{n-1}, \ldots, \epsilon_{1}\right)\right]\right]^{2} \\
& =\left\langle\left(\frac{\left.\ln \mid \Delta_{1, \epsilon_{1}, \ldots, \epsilon_{n-1}}^{(n)}\right)^{2}}{n}\right\rangle_{\nu_{\beta}^{(n)}}-\left(\left\langle\frac{\ln \left|\Delta_{1, \epsilon_{1}, \ldots, \epsilon_{n-1}}^{(n)}\right|}{n}\right\rangle_{\nu_{\beta}^{(n)}}\right)^{2}+O\left(\frac{1}{n}\right),\right.
\end{aligned}
$$


we conclude that $F(\beta)$ is a monotone decreasing convex function. We shall also use the spectral representation for the free energy. Consider the transfer-matrix operator $\mathcal{L}(\beta)$ :

$$
\mathcal{L}(\beta) h\left(\epsilon^{(1)}, \epsilon^{(2)}, \ldots, \epsilon^{(n)}, \ldots\right)=\sum_{\epsilon^{(0)}=0,1} \exp \left[\beta U\left(\epsilon^{(0)}, \epsilon^{(1)}, \epsilon^{(2)}, \ldots, \epsilon^{(n)}, \ldots\right)\right] h\left(\epsilon^{(0)}, \epsilon^{(1)}, \epsilon^{(2)}, \ldots, \epsilon^{(n)}, \ldots\right) .
$$

Since $\mathcal{L}(\beta)$ is a positive linear operator, its largest eigenvalue $\lambda(\beta)$ is strictly positive and simple. It is easy to see that

$$
F(\beta)=\ln \lambda(\beta) .
$$

For an arbitrary point $x \in A_{+}$, denote by $\Delta^{(n)}(x)$ the interval of the $n$th level which contains $x$. It follows from (17) that for points $x$ which are typical with respect to $\mu_{\beta}$ (that is corresponding to a set of full $\mu_{\beta}$-measure.)

$$
\left|\Delta^{(n)}(x)\right| \sim \exp \left[F^{\prime}(\beta) n\right]
$$

More precisely, for $\mu_{\beta}$-almost all $x \in A$

$$
\lim _{n \rightarrow \infty} \frac{\ln \left|\Delta^{(n)}(x)\right|}{n}=F^{\prime}(\beta) .
$$

We next find the total number $N_{n}(\beta)$ of the intervals of $n$-th level whose length is of the order $\left|\Delta^{(n)}(\beta)\right|=\exp \left[F^{\prime}(\beta) n\right]$. We have

$$
N_{n}(\beta)\left|\Delta^{(n)}(\beta)\right|^{\beta} \sim N_{n}(\beta) \exp \left[\beta F^{\prime}(\beta) n\right] \sim Z_{n}(\beta) \sim \exp [F(\beta) n]
$$

which gives

$$
N_{n}(\beta) \sim \exp \left[\left(F(\beta)-\beta F^{\prime}(\beta)\right) n\right] .
$$

Using (24) we can find the Hausdorff dimension $d_{H}(\beta)$ of the set of points $x \in A_{+}$which are typical with respect to the measure $\mu_{\beta}$. Since

$$
N_{n}(\beta)\left|\Delta^{(n)}(\beta)\right|^{d_{H}(\beta)} \sim \exp \left[\left(F(\beta)-\beta F^{\prime}(\beta)+d_{H}(\beta) F^{\prime}(\beta)\right) n\right]
$$

we conclude that

$$
F(\beta)-\beta F^{\prime}(\beta)+d_{H}(\beta) F^{\prime}(\beta)=0
$$

which immediately implies

$$
d_{H}(\beta)=\frac{\beta F^{\prime}(\beta)-F(\beta)}{F^{\prime}(\beta)}=\beta-\frac{F(\beta)}{F^{\prime}(\beta)} .
$$

The Hausdorff dimension $d_{H}(A)$ of the whole attractor $A$ is equal to the maximum of $d_{H}(\beta)$ over all $\beta \in \mathbb{R}$. Let $\beta_{0}$ be the unique solution of the equation $F(\beta)=0$. It is easy to see that $d_{H}(A)=d_{H}\left(\beta_{0}\right)=\beta_{0}$.

We next discuss multifractal properties associated with the Hölder exponents. Consider the integral $u(x)$ of the invariant measure $\mu_{0}$, defined by (3), which is plotted in Fig. 2. The attractor being topologically a Cantor set, $u(x)$ is a variant of the Devil's staircase (see Ref. [1], Section 8.2 ). 


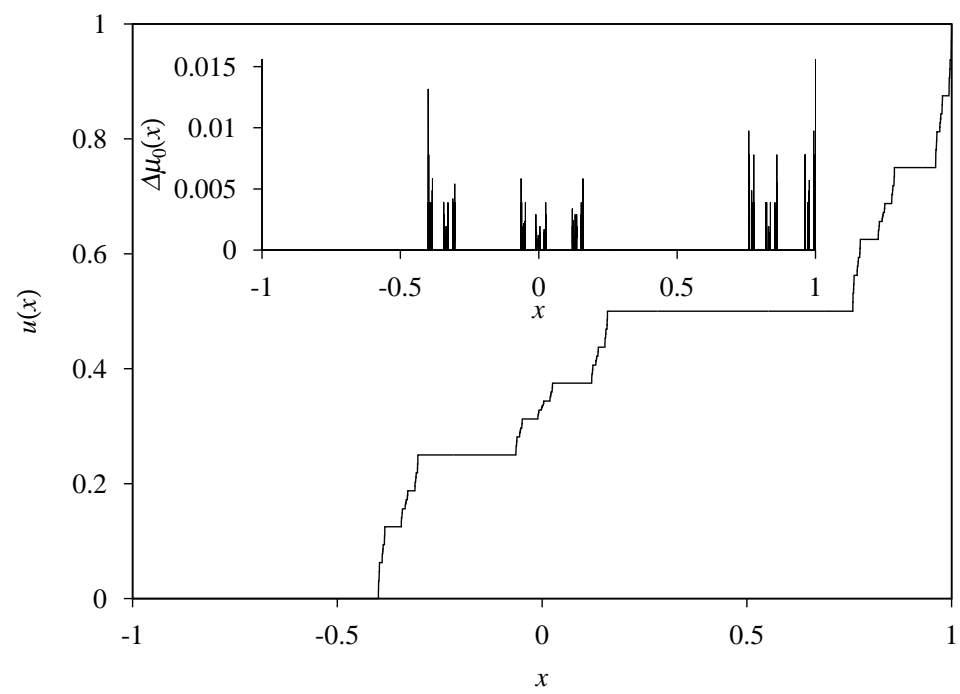

Figure 2: The integral $u(x)$ of the Feigenbaum invariant measure calculated with $2^{15}$ bins of uniform length $\Delta=2^{-14}$ in $[-1,1]$. Inset: The invariant measure smoothed over the distance $\Delta$ calculated as a frequency histogram.

To find its spectrum of Hölder exponents, notice that for every interval $\Delta_{i}^{(n)}$ the increase of $u(x)$ along the interval is equal to $2^{-n}$. Hence $\Delta_{i}^{(n)}$ corresponds to a Hölder exponent $h_{n}(i)=\alpha$ where $\left|\Delta_{i}^{(n)}\right|^{\alpha} \sim \frac{1}{2^{n}}$. This implies

$$
\left|\Delta_{i}^{(n)}\right| \sim 2^{-\frac{n}{\alpha}}
$$

Using (21), (28) we conclude that the Hölder exponent $\alpha$ corresponds to an inverse temperature $\beta(\alpha)$ such that

$$
F^{\prime}(\beta(\alpha))=-\frac{\ln 2}{\alpha}
$$

This gives

$$
\beta(\alpha)=\left(F^{\prime}\right)^{-1}\left(-\frac{\ln 2}{\alpha}\right),
$$

where $\left(F^{\prime}\right)^{-1}(y)$ is the inverse function to $F^{\prime}(x)$. We can now find the Hausdorff dimension $d_{H}(\alpha)$ of the set of points $x \in A$ for which the Hölder exponent of $u(x)$ is equal to $\alpha$ :

$$
d_{H}(\alpha)=d_{H}(\beta(\alpha))=\beta(\alpha)-\frac{F(\beta(\alpha))}{F^{\prime}(\beta(\alpha))} .
$$

Notice that the analysis presented above can be made completely rigorous (see, for example, Refs. [6, 12]).

\subsection{Connection between thermodynamic formalism and standard multifractal analysis}

It is quite interesting to compare the multifractal analysis which we presented above with the one introduced in Ref. [9]. Although we restrict ourselves here to the case of the Feigenbaum attractor, 
the result presented below holds in a much more general setting. Basically our analysis is valid whenever the system under consideration can be described with the help of the thermodynamic formalism.

The basic object for our analysis is the Feigenbaum attractor itself and the method is based on the construction of the thermodynamic formalism for the lengths of the elements of dynamic partitions $\Delta_{i}^{(n)}$. The thermodynamic formalism uses considerable dynamical information about the map $g$. In contrast, the analysis in Ref. [9 is carried out for fractal measures and does not directly use the dynamical information about the system. In the period-doubling setting the fractal measure is $\mu_{0}$. It is the unique invariant measure for $g$ acting on $A$ (see Ref. [6]). It also can be considered as a physical or Sinai-Ruelle-Bowen (SRB) measure on $[-1,1]$. This means that under dynamics given by the map $g$ any initial absolutely continuous distribution $\bar{\mu}$ on $[-1,1]$ converges to $\mu_{0}: g^{(n)}(\bar{\mu}) \rightarrow \mu_{0}$ as $n \rightarrow \infty$. The multifractal analysis in Ref. 9] is based on a function $D_{p}$ which can be defined in the following way. Consider a partition of the interval $[-1,1]$ into subintervals $I_{j}, 1 \leq j \leq N$ of length $l$. Then

$$
D_{p}=\lim _{l \rightarrow 0}\left(\frac{1}{p-1} \frac{\ln \sum_{j=1}^{N}\left(\mu_{0}\left(I_{j}\right)\right)^{p}}{\ln l}\right)=\frac{\alpha}{\ln 2} F(\beta(\alpha)) .
$$

It follows from (32) that

$$
\sum_{j=1}^{N}\left(\mu_{0}\left(I_{j}\right)\right)^{p} \sim N^{D_{p}(1-p)}
$$

Another characteristic of a multifractal measure is given by its spectrum of dimensions $f(\alpha)$ which is just the Legendre transform of $D_{p}(p-1)$ :

$$
f(\alpha)=\inf _{p}\left[\alpha p-D_{p}(p-1)\right] .
$$

The dual Legendre relation allows one to find $D_{p}(p-1)$ from $f(\alpha)$ :

$$
D_{p}(p-1)=\inf _{\alpha}[\alpha p-f(\alpha)] .
$$

We next find a correspondence between the pair $\left(D_{p}(p-1), f(\alpha)\right)$ and the pair of thermodynamic functions $\left(F(\beta), d_{H}(\alpha)\right)$. We shall show that

$$
D_{p}(p-1)=-F^{-1}(p \ln 2), \quad f(\alpha)=d_{H}(\alpha),
$$

where $F^{-1}$ is an inverse function to the free energy $F(\beta)$. To derive the first relation we consider the dynamical partition $\Delta_{i}^{(n)}, 0 \leq i \leq 2^{n}-1$ and assume that $n \gg 1$ but $l \ll \min _{i}\left|\Delta_{i}^{(n)}\right|$. For each $\Delta_{i}^{(n)}$ define

$$
M\left(\Delta_{i}^{(n)}\right)=\sum_{j: I_{j} \subset \Delta_{i}^{(n)}}\left(\mu_{0}\left(I_{j}\right)\right)^{p} .
$$

Notice that the asymptotic behavior of $M\left(\Delta_{i}^{(n)}\right)$ depends only on asymptotic scalings of smaller elements of the dynamical partitions $\Delta_{s}^{(n+m)}$ inside $\Delta_{i}^{(n)}$. The thermodynamic formalism constructed above implies that asymptotically those scalings are completely determined by the potential $U$ and hence they do not depend on $i$. Rescaling the invariant measure inside $\Delta_{i}^{(n)}$ by a 
factor $2^{n}$ we conclude that

$$
M\left(\Delta_{i}^{(n)}\right)=\frac{1}{2^{n p}} \sum_{j: I_{j} \subset \Delta_{i}^{(n)}}\left(2^{n} \mu_{0}\left(I_{j}\right)\right)^{p} \sim \frac{1}{2^{n p}} N_{i}^{D_{q}(1-p)},
$$

where $N_{i}$ is a total number of the intervals $I_{j}$ inside $\Delta_{i}^{(n)}$. Taking the sum over $i$ and using (14) we have

$$
\begin{aligned}
\sum_{j=1}^{N}\left(\mu_{0}\left(I_{j}\right)\right)^{p}=\sum_{0 \leq i \leq 2^{n}-1} M\left(\Delta_{i}^{(n)}\right) & \sim \frac{1}{2^{n p}} \sum_{0 \leq i \leq 2^{n}-1} N_{i}^{D_{p}(1-p)} \sim \frac{1}{2^{n p}} \sum_{0 \leq i \leq 2^{n}-1}\left(\frac{\left|\Delta_{i}^{(n)}\right|}{l}\right)^{D_{p}(1-p)} \\
& \sim \frac{1}{2^{n p}} N^{D_{p}(1-p)} \exp \left[n F\left(D_{p}(1-p)\right)\right]
\end{aligned}
$$

This together with (33) immediately gives

$$
\exp \left[F\left(D_{p}(1-p)\right)\right]=2^{p}
$$

which implies the first relation in (36). We next show that the second relation holds. Using (34) we have

$$
\begin{aligned}
f(\alpha) & =\inf _{p}\left[\alpha p-D_{p}(p-1)\right]=\inf _{p}\left[\alpha p-\left(-F^{-1}(p \ln 2)\right]=\inf _{z}\left[\frac{\alpha}{\ln 2} z+F^{-1}(z)\right]\right. \\
& =\inf _{\beta}\left[\frac{\alpha}{\ln 2} F(\beta)+\beta\right]=\frac{\alpha}{\ln 2} \inf _{\beta}\left[\frac{\ln 2}{\alpha} \beta+F(\beta)\right] .
\end{aligned}
$$

It is easy to see that the extremum in (41) corresponds to $\beta(\alpha)=\left(F^{\prime}\right)^{-1}\left(-\frac{\ln 2}{\alpha}\right)$ which implies

$$
f(\alpha)=\beta(\alpha)+\frac{\alpha}{\ln 2} F(\beta(\alpha))=d_{H}(\alpha) .
$$

Finally we express the scaling exponents $\zeta_{p}$ for the structure functions through the thermodynamic characteristics. The exponent $\zeta_{p}$ is defined by the scaling relation

$$
\left\langle|u(x+l)-u(x)|^{p}\right\rangle \sim l^{\zeta_{p}}
$$

in terms of the integral $u(x)$ of the invariant measure. Let $I_{j}, 1 \leq j \leq N=\frac{2}{l}$ be a partition of $[-1,1]$ into intervals of length $l$. Then

$$
\left\langle|u(x+l)-u(x)|^{p}\right\rangle=\frac{1}{N} \sum_{j=1}^{N}\left(\mu_{0}\left(I_{j}\right)\right)^{p} \sim l N^{D_{p}(1-p)} \sim l^{1+D_{p}(p-1)}
$$

which together with (36) gives

$$
\zeta_{p}=1+D_{p}(p-1)=1-F^{-1}(p \ln 2) .
$$

Using (35) one can also write $\zeta_{p}$ in the following form:

$$
\zeta_{p}=1+\inf _{\alpha}[\alpha p-f(\alpha)]=1+\inf _{\alpha}\left[\alpha p-d_{H}(\alpha)\right]
$$


At $\alpha=\alpha_{\star}$ the infimum (46) is attained, we can then write the relation between $p$ and $\alpha_{\star}$ as

$$
p=\left.d_{H}^{\prime}(\alpha)\right|_{\alpha=\alpha_{\star}}=\frac{F\left(\beta\left(\alpha_{\star}\right)\right)}{\ln 2} .
$$

The scaling exponents for the structure functions are hence obtained as

$$
\zeta_{p}=1-\beta\left(\alpha_{\star}\right)
$$

We now turn to concrete calculations for the scheme presented above. It is easy to see that all the thermodynamic functions can be effectively approximated numerically. The first step is to find approximations for the thermodynamic potential $U\left(\epsilon^{(1)}, \epsilon^{(2)}, \ldots, \epsilon^{(n)}, \ldots\right)$. We shall use Markov approximations $U_{k}\left(\epsilon^{(1)}, \epsilon^{(2)}, \ldots, \epsilon^{(k)}\right)$ which are defined by the following formula:

$$
U_{k}\left(\epsilon^{(1)}, \epsilon^{(2)}, \ldots, \epsilon^{(k)}\right)=\lim _{n \rightarrow \infty} \ln \frac{\left|\Delta_{1,0, \ldots, 0, \epsilon^{(k)}, \epsilon^{(k-1), \ldots, \epsilon^{(1)}}}^{(n)}\right|}{\mid \Delta_{1,0, \ldots, 0, \epsilon^{(k)}, \epsilon^{(k-1)}, \ldots, \epsilon^{(2)}}^{(n-1)}} .
$$

It was shown in [6] that the limit in (49) exists and

$$
\left|U\left(\epsilon^{(1)}, \epsilon^{(2)}, \ldots, \epsilon^{(k)}, \epsilon^{(k+1)}, \ldots\right)-U_{k}\left(\epsilon^{(1)}, \epsilon^{(2)}, \ldots, \epsilon^{(k)}\right)\right| \leq C(2 \gamma)^{k} .
$$

Using the approximate potential $U_{k}$ we can construct a Markov approximation for the transfermatrix operator $\mathcal{L}(\beta)$. Namely, we define a finite dimensional linear operator $\mathcal{L}_{k}(\beta)$ :

$$
\mathcal{L}_{k}(\beta) h\left(\epsilon^{(1)}, \epsilon^{(2)}, \ldots, \epsilon^{(k)}\right)=\sum_{\epsilon^{(0)}=0,1} \exp \left[\beta U_{k+1}\left(\epsilon^{(0)}, \epsilon^{(1)}, \epsilon^{(2)}, \ldots, \epsilon^{(k)}\right)\right] h\left(\epsilon^{(0)}, \epsilon^{(1)}, \epsilon^{(2)}, \ldots, \epsilon^{(k-1)}\right) .
$$

In the matrix representation the operator $\mathcal{L}_{k}(\beta)$ corresponds to a certain $2^{k} \times 2^{k}$ matrix. Denote by $\lambda_{K}(\beta)$ its largest eigenvalue. Then

$$
F_{k}(\beta)=\ln \lambda_{k}(\beta)
$$

is a natural approximation for the free energy $F(\beta)$. It follows from (50) that $F_{k}(\beta)$ converges to $F(\beta)$ exponentially fast in $C^{\infty}$ topology. Using $F_{k}(\beta)$ we can effectively approximate all the multifractal functions which we discussed above. The corresponding numerical results are presented in the next section.

\subsection{Numerical calculation of the free energy and the scaling exponents}

Here we show how to construct the transfer-matrix operator $\mathcal{L}_{k}$ starting from $k=0$ to general $k$. For $k=0$, the matrix operator $\mathcal{L}_{0}$ is just a scalar. Denoting $\Delta_{\epsilon_{1}, \ldots, \epsilon_{n}}^{(n)}$ by $\Delta^{(n)}\left(\epsilon_{1}, \ldots, \epsilon_{n}\right)$ for clarity, let us consider

$$
\exp \left[U_{1}\left(\epsilon^{(0)}\right)\right]=\lim _{n \rightarrow \infty} \frac{\left|\Delta^{(n)}(1, \overbrace{0, \ldots, 0, \epsilon^{(0)}}^{n-2})\right|}{\left|\Delta^{(n-1)}(1, \underbrace{0, \ldots, 0}_{n-2})\right|},
$$


whose analytical expression is easy to calculate. The 0-th order approximation of the free energy is given by

$$
F_{0}(\beta)=\ln \left[e^{\beta U_{1}(0)}+e^{\beta U_{1}(1)}\right] .
$$

For $\epsilon^{(0)}=0$, since $\Delta_{0}^{(n)}=\left[-\alpha^{-n}, \alpha^{-n}\right]$ and $\Delta_{1}^{(n)}=\left[g\left(\alpha^{-n}\right), 1\right]$, we have

$$
\left|\Delta^{(n)}(1,0, \ldots, 0)\right|=1-g\left(\alpha^{-n}\right)=c \alpha^{-2 n}+O\left(\alpha^{-4 n}\right),
$$

where we use the expansion (5) of $g(x)$. Thus $c=1.527 \ldots$ Therefore, the corresponding component of $\mathcal{L}_{0}(\beta=1)$ is

$$
e^{U_{1}(0)}=\lim _{n \rightarrow \infty} \frac{\left|c \alpha^{-2 n}+O\left(\alpha^{-4 n}\right)\right|}{\left|c \alpha^{-2(n-1)}+O\left(\alpha^{-4(n-1)}\right)\right|}=\alpha^{-2} .
$$

Before considering the second term in the argument of the logarithm in (54), we recall the relation

$$
g^{\left(2^{n}\right)}\left(\alpha^{-n} x\right)=(-1)^{n} \alpha^{-n} g(x),
$$

which played a primary role in the proof of Theorem 4.1. of Ref. [6]. Since $\Delta^{(n)}(1,0, \ldots, 0,1)=$ $\Delta_{1+2^{n-1}}^{(n)}$, in view of (57), we have

$$
\begin{aligned}
\left|\Delta^{(n)}(1,0, \ldots, 0,1)\right| & =\left|g^{\left(1+2^{n-1}\right)}(0)-g^{\left(1+2^{n-1}\right)}\left(\alpha^{-n}\right)\right| \\
& =\left|c \alpha^{-2(n-1)}\left\{\left[g\left(\alpha^{-1}\right)\right]^{2}-1\right\}+O\left(\alpha^{-4(n-1)}\right)\right| .
\end{aligned}
$$

Thus for $\epsilon^{(0)}=1$, (153) can be rewritten as

$$
e^{U_{1}(1)}=\lim _{n \rightarrow \infty} \frac{\left|c \alpha^{-2(n-1)}\left\{\left[g\left(\alpha^{-1}\right)\right]^{2}-1\right\}+O\left(\alpha^{-4(n-1)}\right)\right|}{\left|c \alpha^{-2(n-1)}+O\left(\alpha^{-4(n-1)}\right)\right|}=1-\left[g\left(\alpha^{-1}\right)\right]^{2} .
$$

We now arrive at the expression for the 0-th order approximation of the free energy:

$$
F_{0}(\beta)=\ln \left[\alpha^{-2 \beta}+\left\{1-\left[g\left(\alpha^{-1}\right)\right]^{2}\right\}^{\beta}\right] .
$$

Next, consider the $k=1$ approximation to the transfer matrix $\mathcal{L}_{1}(\beta)$. From (151), it can be written in standard matrix notation as

$$
\left(\begin{array}{ll}
e^{\beta U_{2}(0,0)} & e^{\beta U_{2}(1,0)} \\
e^{\beta U_{2}(0,1)} & e^{\beta U_{2}(1,1)}
\end{array}\right) .
$$

It then follows that the free energy is given by

$$
\begin{aligned}
F_{1}(\beta)= & \ln \left[e^{\beta U_{2}(0,0)}+e^{\beta U_{2}(1,1)}+\sqrt{\left(e^{\beta U_{2}(0,0)}-e^{\beta U_{2}(1,1)}\right)^{2}+4 e^{\beta U_{2}(1,0)} e^{\beta U_{2}(0,1)}}\right] \\
& -\ln 2 .
\end{aligned}
$$

The four components of the transfer matrix require the evaluation of suitable exponential terms, expressible by (49), from

$$
\exp \left[U_{2}\left(\epsilon^{(0)}, \epsilon^{(1)}\right)\right]=\lim _{n \rightarrow \infty} \frac{\left|\Delta^{(n)}(1, \overbrace{0, \ldots, 0}^{n-3}, \epsilon^{(1)}, \epsilon^{(0)})\right|}{\left|\Delta^{(n-1)}(1, \underbrace{0, \ldots, 0}_{n-3}, \epsilon^{(1)})\right|} .
$$


Each of these terms is calculated in the same manner as for the $k=0$ case:

$$
\begin{aligned}
e^{U_{2}(0,0)} & =\lim _{n \rightarrow \infty} \frac{\left|\Delta^{(n)}(1,0, \ldots, 0)\right|}{\left|\Delta^{(n-1)}(1,0, \ldots, 0)\right|}=\alpha^{-2}, \\
e^{U_{2}(1,0)} & =\lim _{n \rightarrow \infty} \frac{\left|\Delta^{(n)}(1,0, \ldots, 0,1)\right|}{\left|\Delta^{(n-1)}(1,0, \ldots, 0)\right|}=[g(0)]^{2}-\left[g\left(\alpha^{-1}\right)\right]^{2} \\
& =1-\left[g\left(\alpha^{-1}\right)\right]^{2}, \\
e^{U_{2}(0,1)} & =\lim _{n \rightarrow \infty} \frac{\left|\Delta^{(n)}(1,0, \ldots, 0,1,0)\right|}{\left|\Delta^{(n-1)}(1,0, \ldots, 0,1)\right|} \\
& =\frac{\left|[g(0)]^{2}-\left[g\left(\alpha^{-2}\right)\right]^{2}\right|}{\left|[g(0)]^{2}-\left[g\left(\alpha^{-1}\right)\right]^{2}\right|}=\frac{1-\left[g\left(\alpha^{-2}\right)\right]^{2}}{1-\left[g\left(\alpha^{-1}\right)\right]^{2}}, \\
e^{U_{2}(1,1)} & =\lim _{n \rightarrow \infty} \frac{\left|\Delta^{(n)}(1,0, \ldots, 0,1,1)\right|}{\left|\Delta^{(n-1)}(1,0, \ldots, 0,1)\right|} \\
& =\frac{\left|\left[g^{(3)}(0)\right]^{2}-\left[g^{(3)}\left(\alpha^{-2}\right)\right]^{2}\right|}{\left|[g(0)]^{2}-\left[g\left(\alpha^{-1}\right)\right]^{2}\right|} .
\end{aligned}
$$

For $k=2$, we just write down the transfer matrix $\mathcal{L}_{2}(\beta)$ :

$$
\left(\begin{array}{cccc}
e^{\beta U_{3}(0,0,0)} & 0 & e^{\beta U_{3}(1,0,0)} & 0 \\
e^{\beta U_{3}(0,0,1)} & 0 & e^{\beta U_{3}(1,0,1)} & 0 \\
0 & e^{\beta U_{3}(0,1,0)} & 0 & e^{\beta U_{3}(1,1,0)} \\
0 & e^{\beta U_{3}(0,1,1)} & 0 & e^{\beta U_{3}(1,1,1)}
\end{array}\right)
$$

Now we are in a position to calculate the transfer matrix $\mathcal{L}_{k}(\beta)$ for general $k$. Let us consider the component for $\beta=1$ :

$$
\begin{aligned}
\exp \left[U_{k+1}\left(\epsilon^{(0)}, \epsilon^{(1)}, \ldots, \epsilon^{(k)}\right)\right] & =\lim _{n \rightarrow \infty} \frac{\left|\Delta^{(n)}(1, \overbrace{0, \ldots, 0}^{n-k-2}, \epsilon^{(k)}, \ldots, \epsilon^{(1)}, \epsilon^{(0)})\right|}{\left|\Delta^{(n-1)}(1, \underbrace{0, \ldots, 0}_{n-k-2}, \epsilon^{(k)}, \ldots, \epsilon^{(1)})\right|} \\
& =\lim _{n \rightarrow \infty} \frac{\left|g\left(\Delta^{(n)}(\overbrace{0, \ldots, 0}^{n-k-2}, \epsilon^{(k)}, \ldots, \epsilon^{(1)}, \epsilon^{(0)})\right)\right|}{\left|g\left(\Delta^{(n-1)}(\underbrace{0, \ldots, 0}_{n-k-2}, \epsilon^{(k)}, \ldots, \epsilon^{(1)})\right)\right|} .
\end{aligned}
$$

Hence it is enough to calculate $\left|\Delta^{(n)}\left(0, \ldots, 0, \epsilon^{(k)}, \ldots, \epsilon^{(1)}, \epsilon^{(0)}\right)\right|$. For this we use the following relation:

$$
\begin{gathered}
\left|\Delta^{(n)}(\underbrace{0, \ldots, 0}_{n-k-2}, \epsilon^{(k)}, \ldots, \epsilon^{(1)}, \epsilon^{(0)})\right| \\
=\left|g^{\left(\epsilon^{(k)} 2^{n-k-1}+\ldots+\epsilon^{(1)} 2^{n-2}+\epsilon^{(0)} 2^{n-1}\right)}(0)-g^{\left(\epsilon^{(k)} 2^{n-k-1}+\ldots+\epsilon^{(1)} 2^{n-2}+\epsilon^{(0)} 2^{n-1}\right)}\left(\alpha^{-n}\right)\right| \\
=\alpha^{-(n-k)}\left|\Delta^{(k+1)}\left(\epsilon^{(k)}, \ldots, \epsilon^{(1)}, \epsilon^{(0)}\right)\right| .
\end{gathered}
$$


By (70) the numerator in (69) is given by

$$
\begin{aligned}
\left|\Delta_{1+j \cdot 2^{n-k-1}}^{(n)}\right| & \left.=\left|\left\{1-c \alpha^{-2(n-k-1)}\left[g^{(j)}(0)\right]^{2}\right\}-\left\{1-c \alpha^{-2(n-k-1)}\left[g^{(j)}\left(\alpha^{-(k+1)}\right)\right]^{2}\right\}+O\left(\alpha^{-4(n-k)}\right)\right|\right\} \\
& =c \alpha^{-2(n-k-1)}\left|\left[g^{(j)}(0)\right]^{2}-\left[g^{(j)}\left(\alpha^{-(k+1)}\right)\right]^{2}\right|+O\left(\alpha^{-4(n-k)}\right) .
\end{aligned}
$$

Therefore the component (69) is expressed as

$$
\exp \left[U_{k+1}\left(\epsilon^{(0)}, \epsilon^{(1)}, \ldots, \epsilon^{(k)}\right)\right]=\left|\frac{\left[g^{(j)}(0)\right]^{2}-\left[g^{(j)}\left(\alpha^{-(k+1)}\right)\right]^{2}}{\left[g^{\left(j^{\prime}\right)}(0)\right]^{2}-\left[g^{\left(j^{\prime}\right)}\left(\alpha^{-k}\right)\right]^{2}}\right|,
$$

where $j=\epsilon^{(k)}+\epsilon^{(k-1)} 2+\ldots+\epsilon^{(1)} 2^{k-1}+\epsilon^{(0)} 2^{k}$ and $j^{\prime}=\epsilon^{(k)}+\epsilon^{(k-1)} 2+\ldots+\epsilon^{(1)} 2^{k-1}$. In other words, if the coordinates of the end points of the intervals are given by $\Delta_{j}^{(k+1)}=\left[a_{j}^{(k+1)}, b_{j}^{(k+1)}\right]$ and $\Delta_{j^{\prime}}^{(k)}=\left[a_{j^{\prime}}^{(k)}, b_{j^{\prime}}^{(k)}\right]$, the component (69) can be rewritten as

$$
\exp \left[U_{k+1}\left(\epsilon^{(0)}, \epsilon^{(1)}, \ldots, \epsilon^{(k)}\right)\right]=\left|\frac{\left(a_{j}^{(k+1)}\right)^{2}-\left(b_{j}^{(k+1)}\right)^{2}}{\left(a_{j^{\prime}}^{(k)}\right)^{2}-\left(b_{j^{\prime}}^{(k)}\right)^{2}}\right| .
$$

If the transfer matrix is written as an ordinary $2^{k} \times 2^{k}$ matrix, it is easily found that the row and columns indices are given by (row, column) $=(p, q)$, where

$$
\begin{aligned}
& \text { (row) } p=1+\epsilon^{(k)}+\epsilon^{(k-1)} 2+\ldots+\epsilon^{(1)} 2^{k-1}, \\
& \text { (column) } q=1+\epsilon^{(k-1)}+\epsilon^{(k-2)} 2+\ldots+\epsilon^{(0)} 2^{k-1} \text {. }
\end{aligned}
$$

For numerical calculation of the functions $F_{k}(\beta)$ and $\zeta_{p}$ associated to $u(x)$, we use the expansion (5) for $g(x)$, as given in Ref. 10]. We include terms up to $x^{32}$. Numerical calculation is done with standard double precision (15 significant digits). For obtaining the largest eigenvalue, we use the power method for matrices [13. The multiplication of the matrix is stopped when the relative error of the most dominant eigenvalue becomes less than $10^{-13}$, thus giving $10^{-13}$ absolute error on the $F(\beta)$ function. An alternative approach, also based on the thermodynamic formalism and yielding 10-digit accuracy, may be found in Ref. 14]. Approximate free energies $F_{k}(\beta)$, with $k$ up to 16 , calculated by the transfer-matrix method are shown in Fig. 3. We note that the $k=0$ approximation (60) already gives a reasonable estimate. The discrepancy of the free energy between various orders of approximations is visible at large $\beta$. However the $\beta>0$ region is irrelevant as far as $\zeta_{p}$ for positive $p$ is concerned (see Eq. (48)). The corresponding $\zeta_{p}(p \geq 0)$ are calculated from the $F_{k}(\beta)$ by (45) for different values of $k$; the results, which hardly depend on $k$, are shown in Fig. प(a). We also determined the structure functions of $u(x)$ with $2^{17}$ uniform bins in $[-1,1]$; they are plotted in Fig. 4(b). The exponents $\zeta_{p}$ are then obtained by a least square fit of the structure functions over the range $2^{-16} \sim 1.5 \times 10^{-6} \leq r \leq 1$. With this number of bins, the quality of the fit begins to somewhat deteriorate beyond $p=4$, but otherwise there is rather good agreement between the two methods of determining $\zeta_{p}$. Note that the " $y$-intercept" of the graph of $\zeta_{p}$, namely $\zeta_{0}$, which is the codimension of the support of the invariant measure $\mu_{0}(d x)$, is positive and its numerical value is slightly under one half [6]. This will be important in the sequel. 


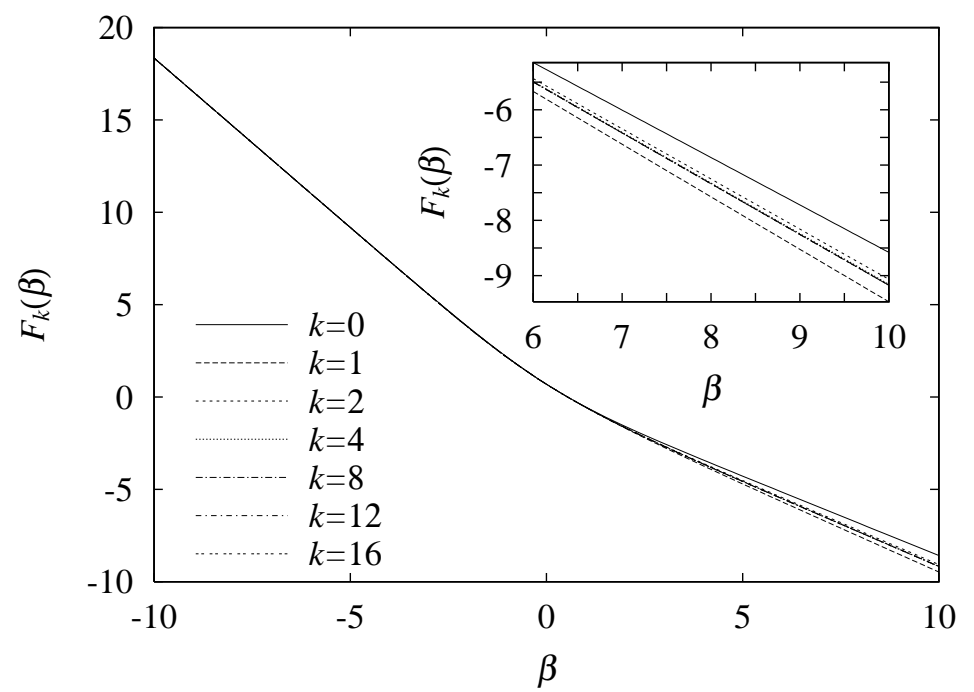

Figure 3: The $k$-th order approximation to the free energy $F_{k}(\beta)$. Inset: Enlargement of the region $6 \leq \beta \leq 10$; when increasing $k$, alternate convergence is observed.
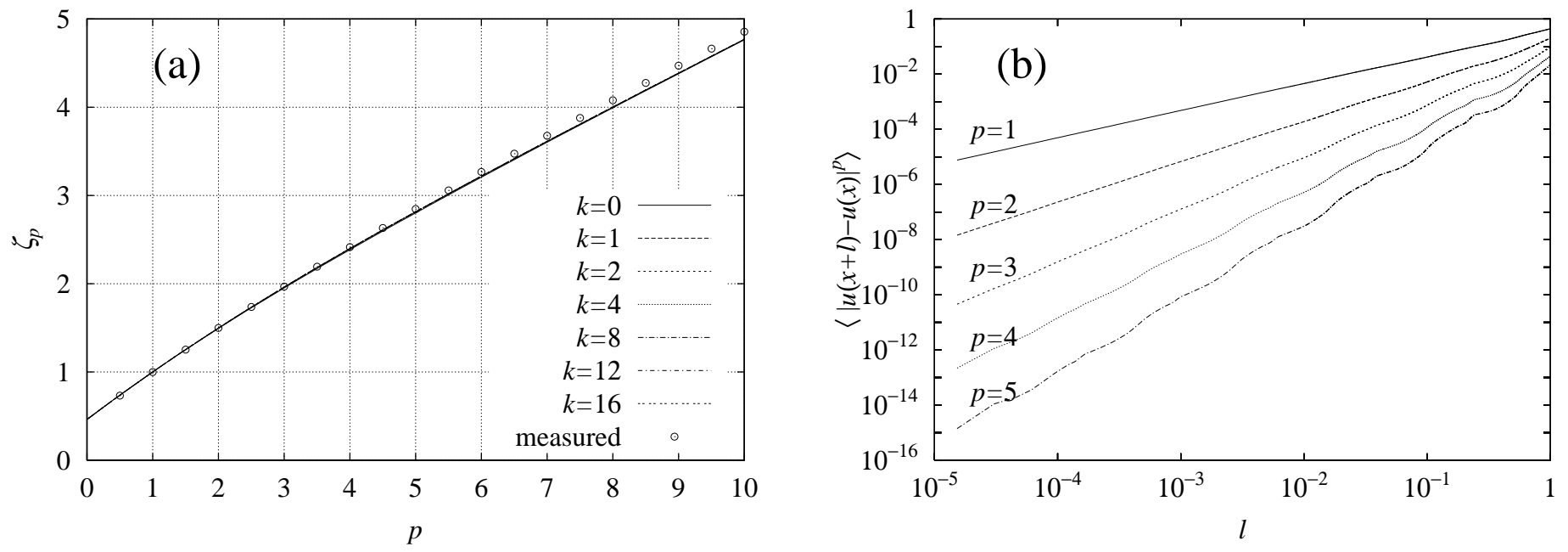

Figure 4: (a) Scaling exponents $\zeta_{p}$ of structure functions obtained by two methods. Open circles: data obtained by a least-square fit of the slopes of the directly measured structure functions shown in (b). Lines: data calculated from the free energy $F_{k}(\beta)$ using (45) (curves for different $k$ are essentially indistinguishable). 


\section{Fractional derivatives for the Feigenbaum attractor}

\subsection{Phenomenology for multifractality and fractional derivatives}

In this section we briefly recall the phenomenological approach to multifractality via fractional derivatives [1] and adapt it to a multifractal measure. We therefore work, not with the measure $\mu_{0}(d x)$ itself, but with its integral $u(x)$. Singularity exponents $\alpha$ may be viewed as local Hölder exponents of $u(x)$, i.e., $|u(x+l)-u(x)| \propto|l|^{\alpha}$ for $(l \rightarrow 0)$. We turn to fractional derivatives of order $a$ defined, as in Ref. [1, as the multiplication in the Fourier space by $\hat{u}(k)$ by $|k|^{a}$ (see Ref. [1] for precise definition). An isolated non-oscillatory singularity with exponent $\alpha$ at a point $x$ implies

$$
\left|D^{a} u(y)\right| \sim|y-x|^{\alpha-a} \quad(y \rightarrow x) .
$$

If $\alpha-a<0$, as we shall assume hereafter, the exponent is negative, the fractional derivative can become arbitrarily large and thus contributes to the tail-behavior of the probability. A key assumption in the phenomenology is that this argument can be carried over to non-isolated multifractal singularities, provided we take all types of singularities into account. For the Feigenbaum invariant measure, we know the Hausdorff dimension $d_{H}(\alpha)$ of the set of points having a singularity with exponent $\alpha$. Assuming that we can also use $d_{H}(\alpha)$ as a covering dimension, we can express the probability to have a singularity of exponent $\alpha$ contributing a fractional derivative of order $a$ which exceeds (in absolute value) a given large value $\xi$, that is we require

$$
|y-x|<\xi^{-\frac{1}{a-\alpha}} .
$$

In terms of the codimension of the set $\mathcal{I}_{\alpha}$, the probability to satisfy (77) is written as

$$
\operatorname{Prob}\left\{\left|D^{a} u\right|>\xi\right\} \propto|y-x|^{d-d_{H}(\alpha)} \propto \xi^{-\frac{d-d_{H}(\alpha)}{a-\alpha}} .
$$

Here $d$ is the spatial dimension $(d=1)$. Taking now into account the singularities with all possible exponents $\alpha$, the tail of the cumulative probability of the fractional derivative of order $a$ is given, to the leading order, by the following power law

$$
\begin{array}{r}
\operatorname{Prob}\left\{\left|D^{a} u\right|>\xi\right\} \propto \xi^{-p_{\star}}, \quad \xi \rightarrow \infty, \\
p_{\star}=\inf _{\alpha<a} \frac{d-d_{H}(\alpha)}{a-\alpha} .
\end{array}
$$

An easy calculation shows that $\alpha_{\star}$ corresponding to the infimum in (80) satisfies

$$
\alpha_{\star}=\alpha_{\star}(a)=a+\frac{d_{H}\left(\alpha_{\star}\right)-d}{d_{H}^{\prime}\left(\alpha_{\star}\right)},
$$

which immediately gives $p_{\star}=d_{H}^{\prime}\left(\alpha_{\star}\right)$. On the other hand, we know that

$$
\zeta_{p}=\inf _{\alpha}\left(p \alpha+d-d_{h}(\alpha)\right) .
$$

Here the infimum is given by an $\alpha$ satisfying the very same relation $p_{\star}=d_{H}^{\prime}\left(\alpha_{\star}\right)$. Hence,

$$
\zeta_{p_{\star}}=p_{\star} \alpha_{\star}+d-d_{H}\left(\alpha_{\star}\right) .
$$


Using (81), we get

$$
\zeta_{p_{\star}}=p_{\star}\left(a+\frac{d_{H}\left(\alpha_{\star}\right)-d}{d_{H}^{\prime}\left(\alpha_{\star}\right)}\right)+d-d_{H}\left(\alpha_{\star}\right)=p_{\star} a,
$$

where the second relation follows from $p_{\star}=d_{H}^{\prime}\left(\alpha_{\star}\right)$. The geometrical interpretation of this equation is that the (negative) exponent of the power-law tail for the fractional derivative of order $a$ is the $p$-value of the intersection of the graph of $\zeta_{p}$ and of a straight line of slope $a$ through the origin.

As shown in Ref. 1], in the presence of the finite range of scaling, the power-law tail (79) emerges only if the multifractality is sufficiently strong. This strength is given by the multifractality parameter $C(a)$, a measure of how strongly the data depart from being self similar (which would imply $\left.\zeta_{p} \propto p\right)$ :

$$
C(a) \equiv a-\alpha_{\star}=\frac{\zeta_{p_{\star}}}{p_{\star}}-\left.\frac{d \zeta_{p}}{d p}\right|_{p=p_{\star}},
$$

where $\alpha_{\star} \equiv d \zeta_{p} /\left.d p\right|_{p=p_{\star}}$. It was shown that observability of the power-law requires a sufficiently large value for the product $n C(a)$, where $n$ is the number of octaves over which the data present multifractal scaling. In practice it was found in Ref. [1] that

$$
n C(a) \geq 10
$$

For example, fully-developed turbulence velocity data [15] have typical $C$ values of the order of $1 / 30$, thereby requiring a monstrous inertial range of about 300 octaves for observability of power-law tails. As we shall see, the situation is much more favorable for the Feigenbaum invariant measure.

Before turning to numerical questions, we comment on an issue raised by an anonymous referee who worried about the nonlocal character of the fractional derivative and wrote in essence that our approach makes sense, strictly speaking, only for (statistically) translationally invariant in space systems: otherwise, if the system consists of components whose "fractal properties" are rather different the results will be smeared out. Our feeling about such matters is summarized as follows. First one can observe that, of course, the attractor for the Feigenbaum map is not homogeneous (translation-invariant) but after zooming in it becomes increasingly so; the fractional derivative is not a local operator but the tail of its PDF is likely to be dominated by strongly localized events. Second, a more technical observation. The idea of the multi-fractal analysis is based on the fact that the dynamics of a system determines a variety of scales. It is important that these scales do not depend on a particular place in the phase space. On the contrary, they are present and "interact" with each other everywhere. In the case of the Feigenbaum attractor the scales depend on a symbolic location in a system of partitions. In physical systems, like homogeneous turbulence, such partitions are difficult to define rigorously. However, the invariance with respect to the space coordinate is still present and forms a basis for applicability of the multifractal calculus.

\subsection{Numerical analysis of fractional derivatives}

The phenomenological arguments presented in the previous section suggest that we should find power-law tails in the cumulative probability for fractional derivatives of $u(x)$ for suitable orders $a$. 
Table 1: For the Feigenbaum invariant measure we show the scaling exponents $\zeta_{p}$, the corresponding inverse temperature $\beta$ 's, the multifractality parameter $C$ and the number of scaling octaves needed.

\begin{tabular}{ccccc}
\hline$p$ & $\zeta_{p}$ & $\beta$ & $C$ & number of octaves needed \\
\hline 1.0 & 1.0 & 0.0 & 0.48 & 21 \\
\hline 1.5 & 1.2540292658 & -2.54029265895 & 0.34 & 29 \\
\hline 2.0 & 1.4985620106 & -4.98562010659 & 0.28 & 36 \\
\hline 2.5 & 1.7344372955 & -7.34437295506 & 0.24 & 42 \\
\hline 3.0 & 1.9625763533 & -9.62576353333 & 0.22 & 45 \\
\hline
\end{tabular}

Inspection of Fig. 团indicates that $a$ should be between the minimum slope of the graph and unity. The value $a=1$ is of course not a fractional order but, as we shall see, it is associated with a powerlaw tail of exponent minus one. ${ }^{1}$ The minimum slope can be easily found. Indeed, $F(\beta)$ takes large values when $\beta$ is large negative. In this case the main contribution to $F(\beta)$ comes from the shortest interval of the partition with the length of the order of $\gamma^{2 n}$. Hence, $F(\beta) /|\beta| \rightarrow-2 \ln \gamma$ in the limit $\beta \rightarrow-\infty$. This gives the following lower bound of the differentiation order:

$$
\lim _{p \rightarrow \infty} \frac{\zeta_{p}}{p}=\lim _{p \rightarrow \infty} \frac{1-F^{-1}(p \ln 2)}{p}=-\frac{\ln 2}{2 \ln \gamma} \approx 0.3777 .
$$

We have already observed that, because the $\zeta_{p}$ graph does not pass through the origin, substantial values can be expected for the multifractality parameter $C(a)$. The actual values of $C$, associated to values of $p$ ranging from 1 to 3 by increments of 0.5 are shown in Table 1 together with the number $n$ of scaling octaves needed determined by $n C \approx 10$ (cf. Eq. (866) ). In practice, on a 32 bit machine, we are limited to about 25 octaves of dynamical range in resolution over the interval $[-1,1]$. This should be enough to observe power-law tails.

Indeed, Fig. 5 shows five instances of cumulative probabilities of fractional derivatives with power-law tails, corresponding to the values of the exponent $p$ listed in Table1. The corresponding order of differentiation $a$ ranges between 0.65 and $1 .{ }^{2}$ Since the function $u(x)$ which we are analyzing is not periodic, we resort to the Hann windowing technique employed previously in Ref. 1] (Section 13.4). Also, we use rank ordering to avoid binning.

The power-law behavior observed is consistent with the phenomenological theory presented in Section 3.1 the residual discrepancies being due to the resolution of $2^{25}$ bins.

\section{Concluding remarks}

We have found solid numerical evidence for the presence of power-law tails in the cumulative distribution of fractional derivatives for the integral $u(x)$ of the invariant measure of the Feigenbaum map. Furthermore the exponents measured are consistent with those predicted by phenomenological arguments from the spectrum of singularities. Since we have a fairly deep understanding of

\footnotetext{
${ }^{1}$ This is a consequence of $\zeta_{1}=1-F^{-1}(\ln 2)=1$. (Putting $\beta=0$ into the partition function $Z_{k}(\beta) \sim$ $\sum_{j=0}^{2^{k}-1}\left|\Delta_{j}^{(k)}\right|^{\beta}$, we have $F_{k}(0)=\ln Z_{k}(0) / k=\ln 2$.)

${ }^{2}$ As predicted by the theory, when $a$ is too small, e.g. for $a=0$, no power-law tail is observed.
} 

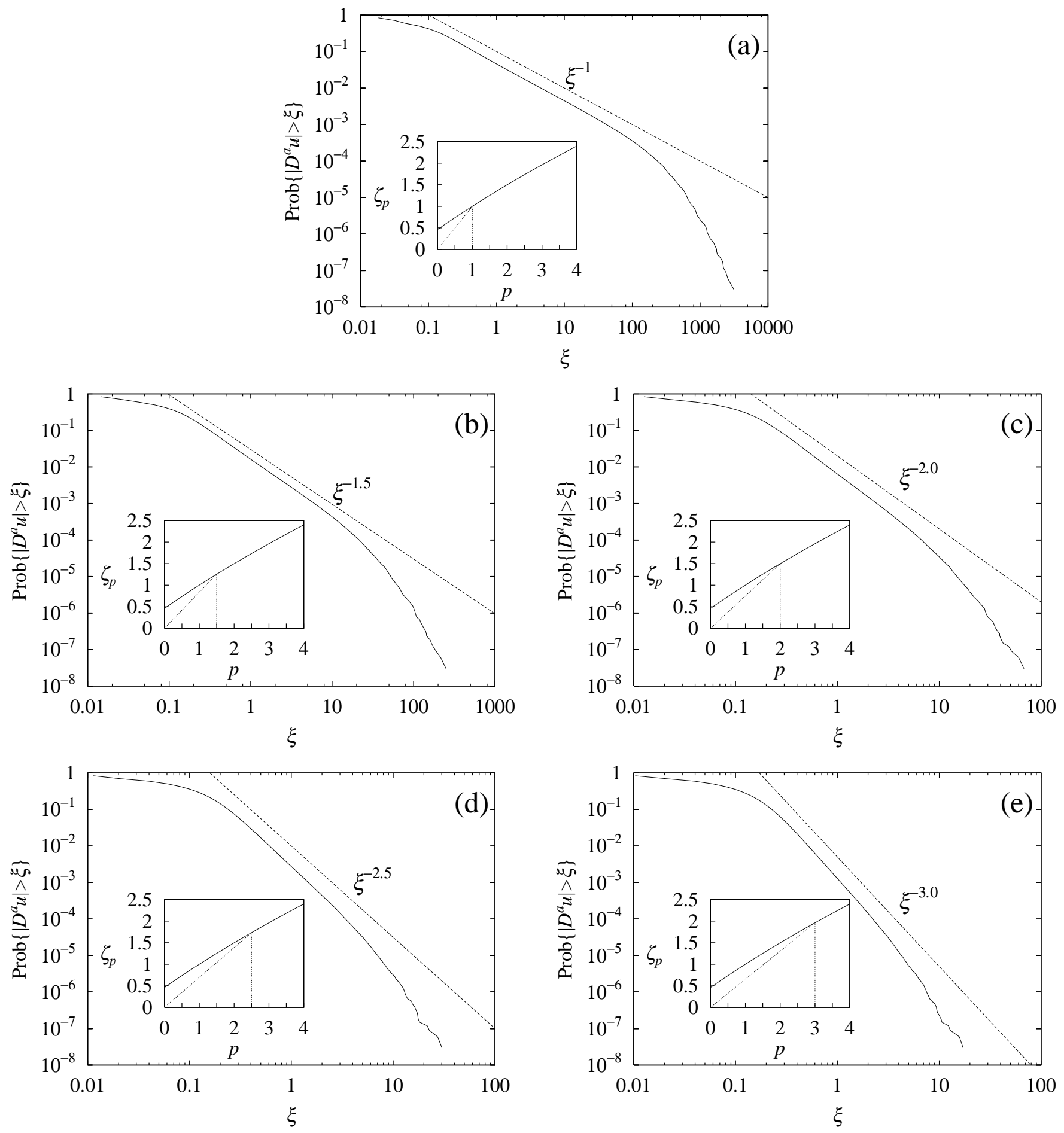

Figure 5: Cumulative probabilities of absolute values of fractional derivatives of various orders $a=\zeta_{p_{\star}} / p_{\star}$ for the Feigenbaum invariant measure. Each function displays a power-law tail with an exponent fairly close to the predicted value $-p_{\star}$. Insets: corresponding $\zeta_{p}$ graphs. (a) $a=1.0\left(p_{\star}=1.0\right)$. (b) $a=0.83\left(p_{\star}=1.5\right)$. (c) $a=0.74\left(p_{\star}=2.0\right) .(\mathrm{d}) a=0.69\left(p_{\star}=2.5\right)$. (e) $a=0.65\left(p_{\star}=3.0\right)$. 
the structure of the attractor, thanks in particular to the thermodynamic formalism, a reasonable goal may be to actually prove the results. The main difficulty is that the operation of fractional derivative is non-local. However, we believe that a rigorous analysis here is still possible due to the quite simple spectral structure of the dynamical system corresponding to the Feigenbaum attractor.

\section{Acknowledgments}

We are grateful to Rahul Pandit for useful remarks. Computational resources were provided by the Yukawa Institute (Kyoto). This research was supported by the European Union under contract HPRN-CT-2000-00162 and by the Indo-French Centre for the Promotion of Advanced Research (IFCPAR 2404-2).

\section{References}

[1] U. Frisch and T. Matsumoto, On multifractality and fractional derivatives, J. Stat. Phys. 108:1181-1202 (2002).

[2] R. Benzi, L. Biferale, A. Crisanti, G. Paladin, M. Vergassola and A. Vulpiani, A random process for the construction of multiaffine fields, Physica D 65:163-171 (1993).

[3] H. Kesten, Random difference equations and renewal theory for products of random matrices, Acta. Math. 131:207-248 (1973).

[4] M.J. Feigenbaum, Quantitative universality for a class of nonlinear transformations, J. Stat. Phys. 19:25-52 (1978).

[5] M.J. Feigenbaum, The transition to aperiodic behavior in turbulent systems, Comm. Math. Phys. 77:65-86 (1980).

[6] E.B. Vul, Ya.G. Sinai and K.M. Khanin, Feigenbaum universality and the thermodynamic formalism, Russian Math. Surveys 39:1-40 (1984).

[7] G. Parisi and U. Frisch, On the singularity structure of fully developed turbulence, in Turbulence and Predictability in Geophysical Fluid Dynamics, Proceedings of International School of Physics 'Enrico Fermi', Jun. 14-24 1983, Varenna, Italy, M. Ghil, R. Benzi and G. Parisi, eds., pp. 84-87, North Holland (1985).

[8] R. Benzi, G. Paladin, G. Parisi and A. Vulpiani, On the multifractal nature of fully developed turbulence and chaotic systems J. Phys. A17:3521-3531 (1984).

[9] T.C. Halsey, M.H. Jensen, L.P. Kadanoff, I. Procaccia and B.I. Shraiman, Fractal measures and their singularities: the characterization of strange sets, Phys. Rev. A 33:1141-1151 (1986).

[10] O.E. Lanford, A computer-assisted proof of the Feigenbaum conjectures, Bull. Ame. Math. Soc. 6:427-434 (1982). 
[11] U. Frisch, Turbulence, the Legacy of A. N. Kolmogorov, Cambridge University Press, Cambridge (1995).

[12] P. Collet, J.L. Lebowitz and A. Porzio, The dimension spectrum of some dynamical systems, J. Stat. Phys. 47:609-644 (1987).

[13] G.H. Golub and C.F. Van Loan, Matrix Computations 3rd edition, The Johns Hopkins University Press, Baltimore (1996), Section 7.3.

[14] Z. Kovács, Universal $f(\alpha)$ spectrum as an eigenvalue, J. Phys. A 22:5161-5165 (1989).

[15] F. Anselmet, Y. Gagne, E.J. Hopfinger and R.A. Antonia, High-order velocity structure functions in turbulent shear flow, J. Fluid Mech. 140:63-89 (1984). 